\title{
Slow and Steady: A Systematic Review of ICU Care Models Relevant to Pediatric Chronic Critical IIIness
}

\author{
Rachel Troch ${ }^{1}$ Jamie Schwartz ${ }^{2}$ Renee Boss ${ }^{2,3}$ \\ ${ }^{1}$ Department of Neonatology, Children's National Hospital, \\ Washington, District of Columbia, United States \\ 2 Department of Ananthesiology and Critical Care Medicine, Johns Hopkins \\ University School of Medicine, Baltimore, Maryland, United States \\ ${ }^{3}$ Department of Neonatal - Perinatal Medicine, Berman Institute of \\ Bioethics, Baltimore, Maryland, United States \\ J Pediatr Intensive Care 2020;9:233-240.
}

\begin{abstract}
Address for correspondence Rachel Troch, MD, Department of Neonatology, Children's National Hospital, 111 Michigan Ave NW, Washington, DC 20010, United States (e-mail: rtroch2@childrensnational.org).
\end{abstract}

\begin{abstract}
Keywords

- chronic critical illness

- intensive care

- multidisciplinary

- protocol

- pathway
\end{abstract}

There is a growing population of children with prolonged intensive care unit (ICU) hospitalization. These children with chronic critical illness (CCI) have a high health care utilization. Emerging data suggest a mismatch between the ICU acute care models and the daily care needs of these patients. Clinicians and parents report that the frequent treatment alterations typical for ICU care may be interrupting and jeopardizing the slow recoveries typical for children with $\mathrm{CCl}$. These frequent treatment titrations could therefore be prolonging ICU stays even further. The aim of this study is to evaluate and summarize existing literature regarding pace and consistency of ICU care for patients with CCl. We performed a systematic review using the Preferred Reporting Items for Systematic Reviews and Meta-Analyses (PRISMA) guidelines (of September 2018). PubMed (biomedical and life sciences literature), Excerpta Medica database (EMBASE), and The Cumulative Index to Nursing and Allied Health Literature (CINAHL) were searched for English-language studies with data about $\mathrm{CCl}$, care models, and pacing of clinical management. Four unique papers were identified. Our most important finding was that quality data on chronic ICU management, particularly for children, is sparse. All papers in this review confirmed the unique needs of chronic patients, particularly related to respiratory management, which is a common driver of ICU length of stay. Taken together, the papers support the hypothesis that protocols to reduce interdisciplinary management variability and to allow for slower management pacing should be studied for their impact on patient and health system outcomes. Optimizing value in ICU care requires mapping of resources to patient needs, particularly for patients with the most intense resource utilization. For children with $\mathrm{CCl}$, parents and clinicians report that rapid treatment changes undermine recovery and prolong ICU stays. This review highlights the lack of quality pediatric research in this area and supports further investigation of a "slow and steady" approach to ICU management for children with CCI. received

February 3, 2020

accepted after revision

May 5, 2020

published online

June 17, 2020
Copyright @ 2020 by Georg Thieme

Verlag KG, Stuttgart · New York
DOI https://doi.org/

10.1055/s-0040-1713160. ISSN $2146-4618$. 


\section{Introduction}

A growing number of neonatal and pediatric intensive care unit (ICU) patients have chronic critical illness (CCI), which is characterized by prolonged hospitalization/s, multiorgan conditions, and dependence on medical technologies. ${ }^{1}$ In the United States, most of such patients are hospitalized in academic medical centers where they can access multiple subspecialists along with facilities and therapies integral to complex care. ${ }^{2,3}$ While the actual number of such patients at any given center may be relatively small, these patients account for a disproportionate number of ICU bed days. ${ }^{2,4}$ After their initial acute stabilization, many days and weeks of their ICU stays involve chronic management of conditions that are stabilized with mechanical ventilation, continuous medication infusions, and other medical equipment or therapies not provided outside of the ICU. Without broader access to non-ICU sites of care, these patients are likely to continue to utilize substantial ICU resources. Such patients are not limited to the United States alone but are admitted to ICUs all around the world. ${ }^{5-7}$

We previously showed that ICU systems are a poor match for patients with $\mathrm{CCI}$ in several ways, including that rotating ICU staff cannot offer continuity to chronic disease management, and subspecialty silos undermine comprehensive goals of care. ${ }^{8}$ Clinicians and parents suggest another important mismatch between chronic CCI needs and the ICU acute care model. Parents often report that their children's ICU stays are prolonged in part because of frequent treatment titrations, separated by too brief assessments of clinical response, without accounting for the slowed homeostasis that children with multiorgan conditions can have. Clinicians also recognize that this acute care treatment approach can thwart the clinical progress or even trigger recurrent setbacks for children with CCI. A "slow and steady" approach to the chronic ICU phase is proposed by both, the parents and the providers, as an alternative model of care. ${ }^{8}$ The slow and steady clinical approach would prioritize paced (i.e., "slow") and consistent (i.e., "steady") clinical management from the entire interdisciplinary team, accepting a slower physiologic trajectory than is historically expected for the ICU patients.

While limited research has targeted ideal clinical approaches to patients with $\mathrm{CCI}$, consensus guidelines for adults with $\mathrm{CCI}$ contain elements that are consistent with a "slow and steady" approach. The American College of Chest Physicians, American Association for Respiratory Care, and the American College of Critical Care Medicine produced the consensus recommendation: "Weaning strategies in the prolonged mechanical ventilation patient should be slow-paced and should include gradually lengthening self-breathing trials"(Rationale and Evidence-Grade C) and suggested the "aggressive ICU mindset" may not be optimal for the "slowly recovering patient." Similarly, the National Association for Medical Direction of Respiratory Care, a physician advocacy group, concludes that the standard ICU time frame may not be appropriate for long-term ventilation (LTV) patients where "recovery is usually much slower than in most acute ICU patients." 10

As we strive to improve management of chronic patients in neonatal and pediatric ICUs, data collection is required to replace management philosophies with evidence-based approaches. We designed this systematic review to summarize existing literature regarding approaches to chronic ICU care delivery, with specific focus on pace of care. The results suggest next steps for clinical and research strategies to address the needs of this specific pediatric population.

\section{Materials and Methods}

Using the Preferred Reporting Items for Systematic Reviews and Meta-Analyses (PRISMA) guidelines, a systematic literature review was completed. Relevant electronic databases, including PubMed (biomedical and life sciences literature), Excerpta Medica database (EMBASE), and The Cumulative Index to Nursing and Allied Health Literature (CINAHL), were searched for English-language studies with data about $\mathrm{CCI}$ and pacing of clinical management from inception to February 2020. The first set of search terms was designed to search for articles related to chronically critically ill patients and the second set of terms focused on care delivery systems related to pace of care for these patients. Search terms were key words related to critical illness, prolonged hospitalization, and pace of care, as listed in - Table 1. CCI for adult patients has previously been defined as those hospitalized at least 8 consecutive days in the ICU with prolonged acute mechanical ventilation, tracheotomy, sepsis, severe wounds, or multiple organ failure. However, no such definition exists for pediatric patients. Previous studies have suggested a focus on underlying conditions and their impact on developmental status, use of medical technology, as well as length of stay (LOS), and recurrent hospitalizations. ${ }^{1}$ Because research in this area is limited, there were no eligibility restrictions on date of study, study design, country of origin, single versus multi-institutional recruitment, or inpatient versus outpatient setting.

The search yielded 12,495 articles from which 1,698 duplicates were removed leaving 10,797 articles. In a preselection process, one author (R.T.) screened titles and abstracts for content with information relevant to pacing and consistency of chronic ICU care. Clearly unrelated article topics were excluded. The topic of excluded articles included areas such as pathology, specific management, palliative care, psychosocial concerns, communication, education, decision making, and rapid response teams (- Table 2, List 1). One hundred ninety-four articles remained. Two authors (R.T. and R.B.) then performed abstract and title screening from which 41 full text articles were identified related to the pacing and consistency of care for $\mathrm{CCI}$ patients. We also hand searched the reference lists of included articles and identified an additional 15 articles. The full text of these 56 articles were evaluated by two authors (R.T. and R.B.) and four unique papers were identified (-Fig. 1). Articles were excluded if they did not focus on CCI patients, or relate to an ICU setting, or pertain to timing or pace of care. Additionally, only original studies were included, consensus guidelines, commentaries, editorials, case reports, conference abstracts, and reviews were excluded (-Table 2, List 2). Data extraction was then performed by three authors (R.T., R.B., and J.S.). Information about study design, sample size, outcomes, and 
Table 1 Databases and search terms

\begin{tabular}{|c|c|}
\hline Database & Search term \\
\hline PubMed & 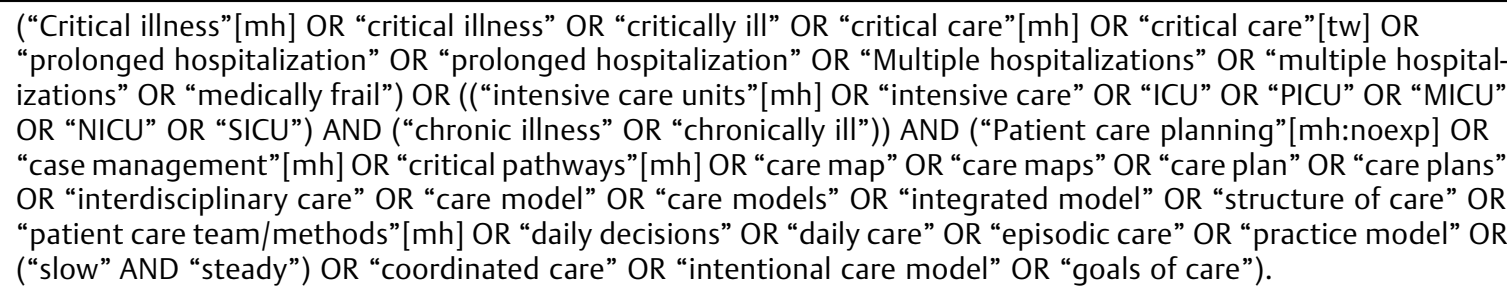 \\
\hline EMBASE & 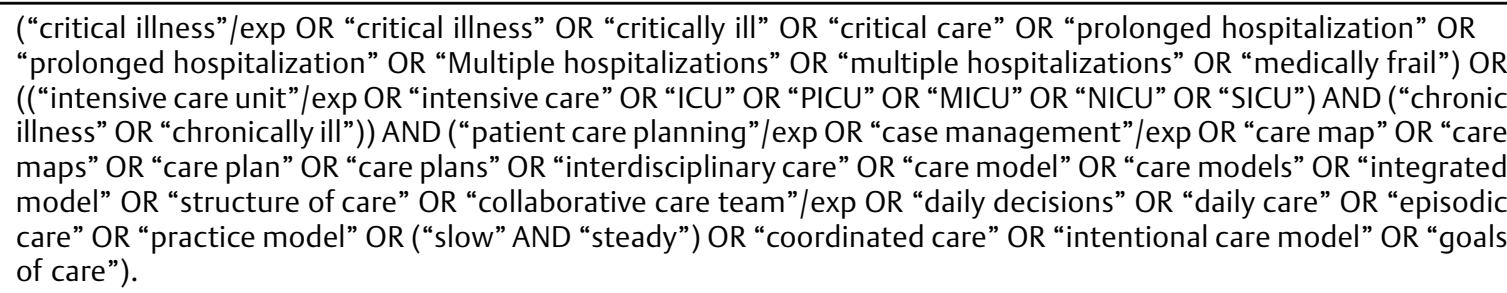 \\
\hline CINAHL & 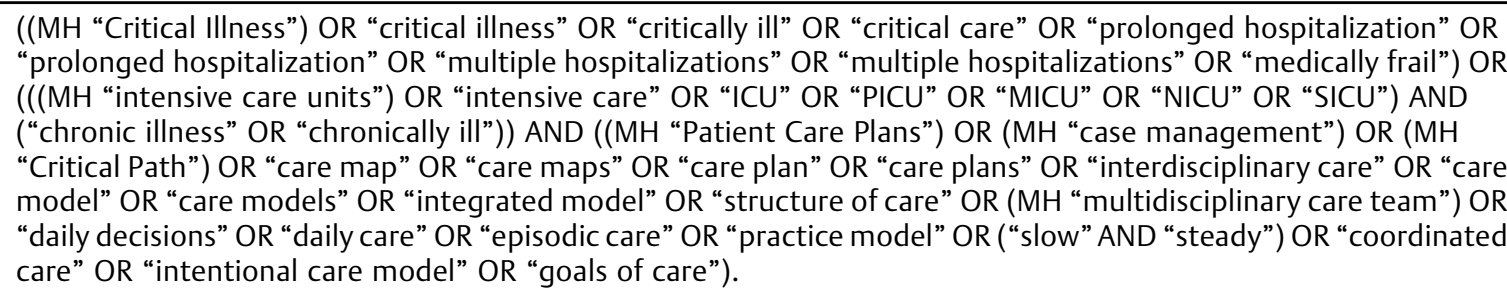 \\
\hline
\end{tabular}

Abbreviations: CINAHL, Cumulative Index to Nursing and Allied Health Literature; EMBASE, Excerpta Medica Database; ICU, intensive care unit; MICU, medical intensive care unit; NICU, neonatal intensive care unit; PICU, pediatric intensive care unit; PubMed, Biomedical and Life Sciences Literature; SICU, surgical intensive care unit.

Table 2 Article exclusion

List 1: Topics of excluded articles in the initial screen

- Pathology specific: COPD, ARDS, delirium, sleep, pain, sepsis, CLABSI, glycemic control, strokes, TBI, wound care, burns, arrythmias, ECMO, transplant, post cardiac surgery, pregnancy, oncology, geriatric frailty.

- Microbiology and antimicrobials.

- Pharmacology.

- Transfusion medicine and anticoagulation.

- Nutrition evaluation and fluid management.

- Trauma and military medicine.

- Transport medicine.

- Palliative care, withdrawal of care, goals of care, and ethics.

- Morbidity, mortality, and survivorship.

- Psychosocial: post intensive care syndrome, family dynamics, parental and family stress, burnout, patient mental health.

- Communication: team communication, bedside rounds, handoffs, transitions of care, social media use, telemedicine, EMR.

- Medical and nursing education and simulation.

- Decision-making: decision tools, checklists, informed consent.

- Rapid response teams and early warning systems.

- Illness severity scores.

- Patient safety.

- Hemodynamic monitoring technology and bedside imaging.

List 2: Reason for article exclusion in the full text evaluation

- Wrong patient population.

- Wrong outcomes.

- Wrong setting.

- Wrong study design.

Abbreviations: ARDS, acute respiratory distress syndrome; CLABSI, central line-associated bloodstream infection; COPD, chronic obstructive pulmonary disease; ECMO, extracorporeal membrane oxygenation; EMR, electronic medical record; TBI, traumatic brain injury. 


\section{PRISMA FLOW DIAGRAM}

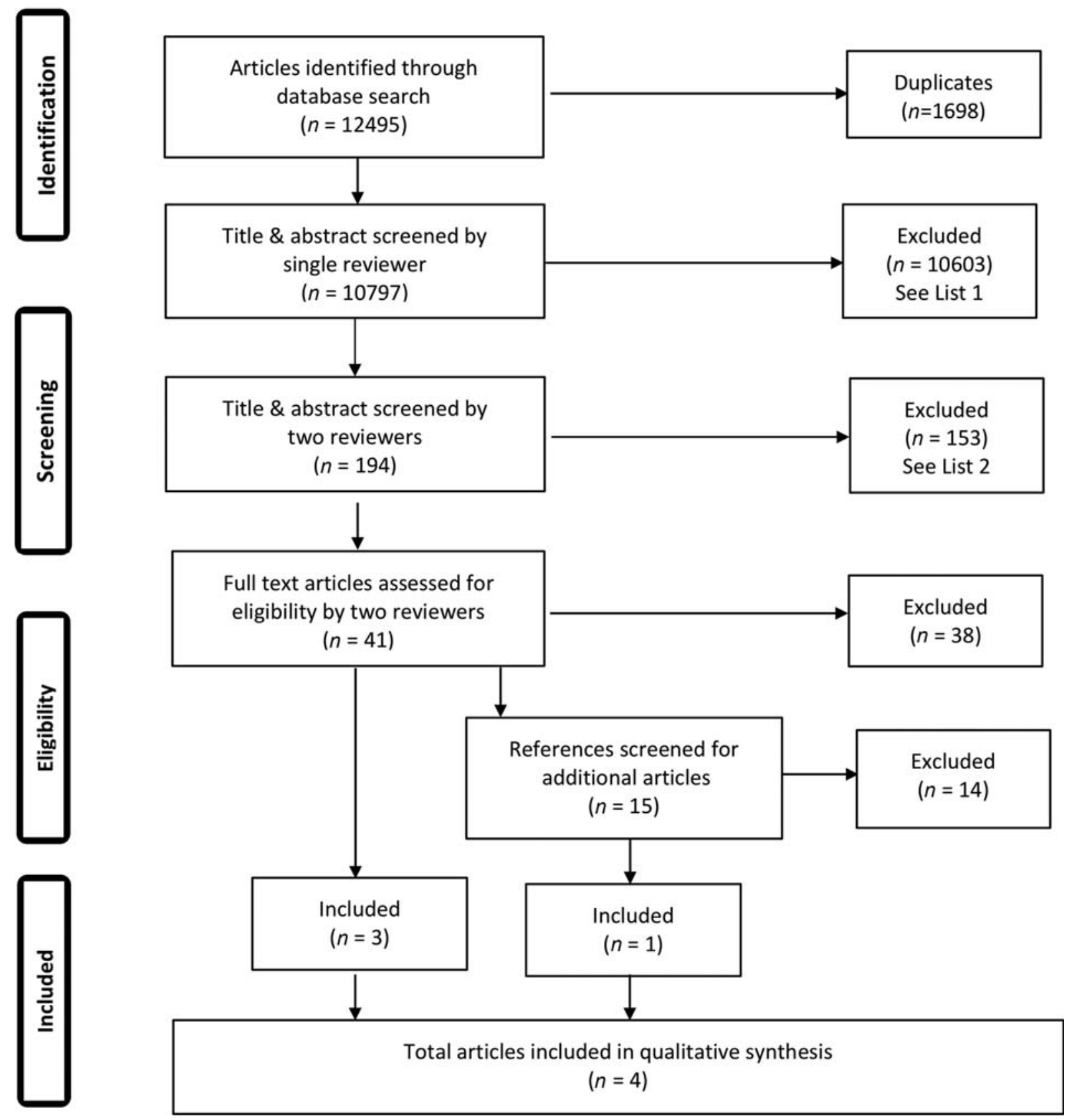

Fig. 1 Preferred Reporting Items for Systematic Reviews and Meta-Analyses (PRIMSA) Diagram. The initial search yielded 6,511 papers. Of these, four unique articles were identified pertaining to chronic ICU care delivery, with specific focus on pace and consistency of care. Exclusion reasons are listed in - Table 2: list 1 and list 2. Figure based off the PRISMA statement (Adapted from Moher et al 2009. ${ }^{28}$ ). ICU, intensive care unit.

specific findings regarding the pace of care were recorded (-Table 3).

\section{Results}

Three of the four final articles were from the United States; all were published between 1991 and 2012. They included two prospective studies, one retrospective study, and one randomized controlled trial ( - Table 3 ). Only one article targeted pediatric patients. None of the articles specifically addressed patients with CCIs, though all included patients receiving LTV who likely met criteria for $\mathrm{CCI}$.

Kun et al authored the only pediatric article. ${ }^{11}$ This retrospective chart review applied a generalized estimate equation to explore risk factors for readmission for patients newly discharged with home mechanical ventilation. They found that $40 \%$ of children discharged on home mechanical ventilation were readmitted within 1 year. A change in ventilator management less than 1 week before discharge was identified as a risk factor for early readmission, 


\begin{tabular}{|c|c|c|c|c|}
\hline 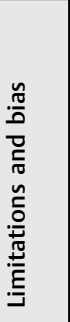 & 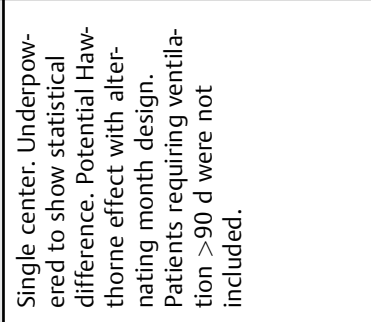 & 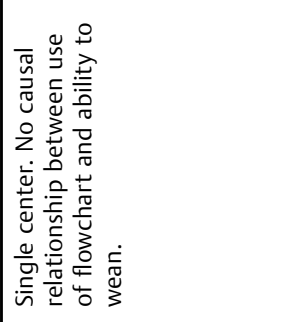 & 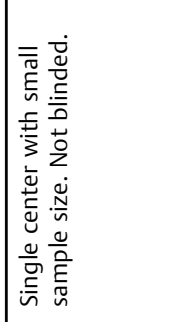 & 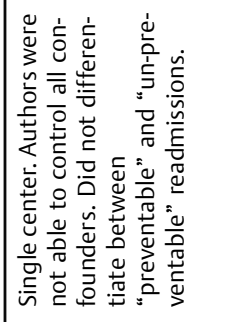 \\
\hline 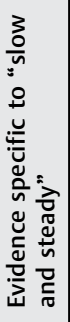 & 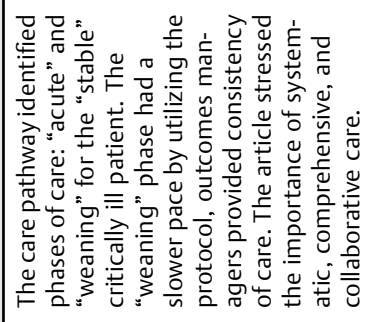 & 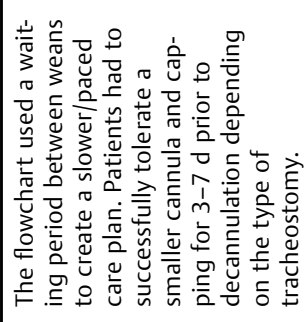 & 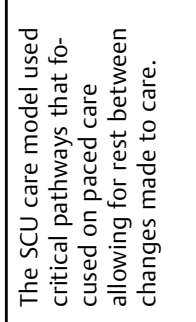 & 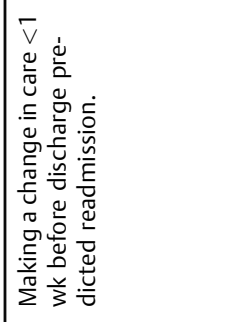 \\
\hline & 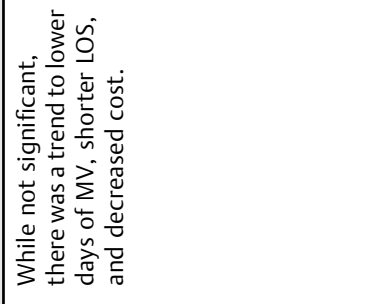 & 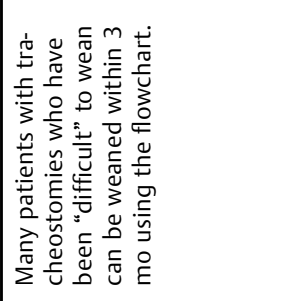 & 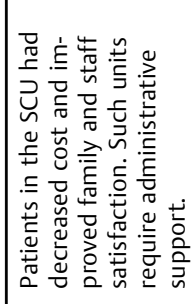 & 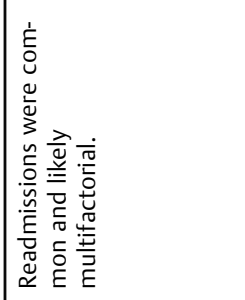 \\
\hline$\stackrel{\check{n}}{\overrightarrow{\underline{y}}}$ & 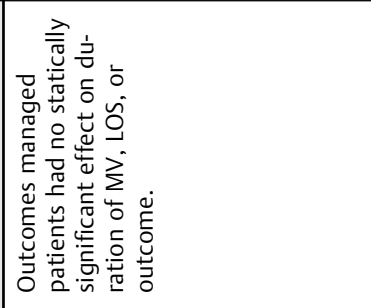 & 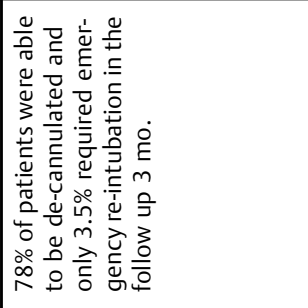 & 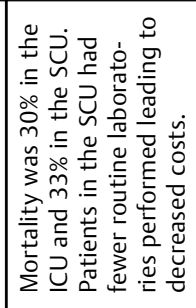 & 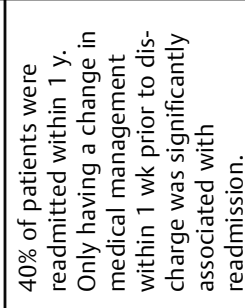 \\
\hline 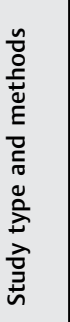 & 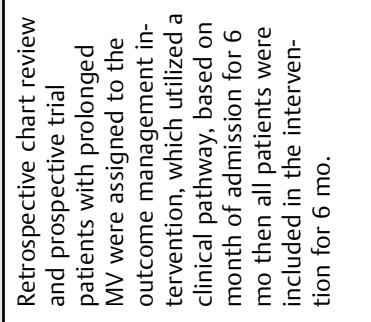 & 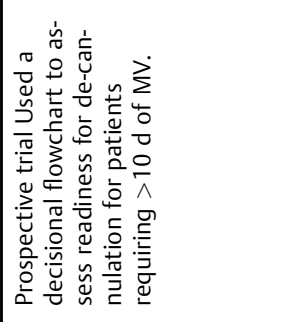 & 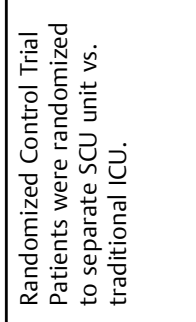 & 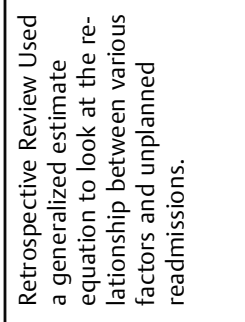 \\
\hline 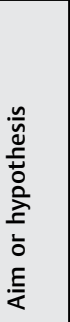 & 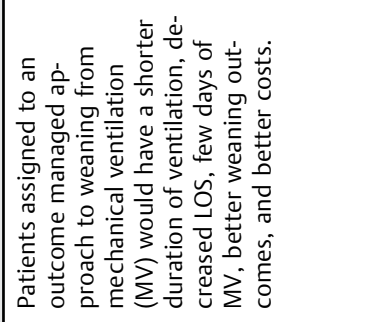 & 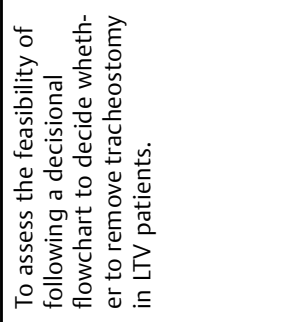 & 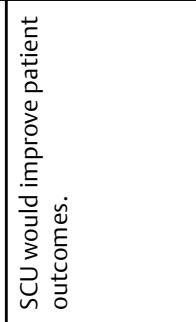 & 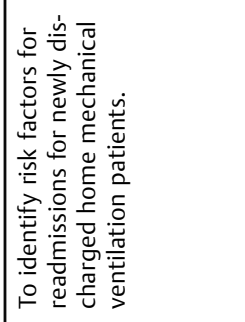 \\
\hline 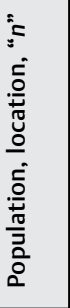 & 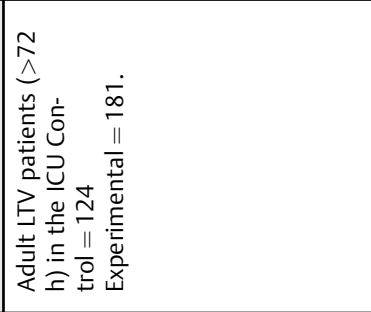 & 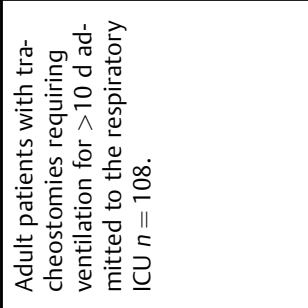 & 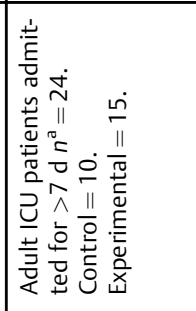 & 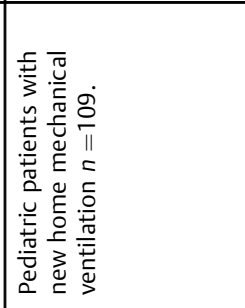 \\
\hline 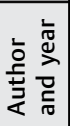 & 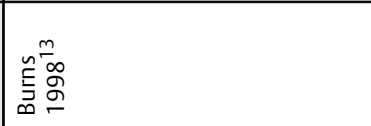 & 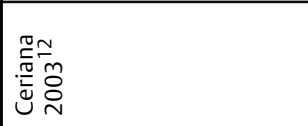 & 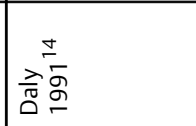 & 产 \\
\hline
\end{tabular}

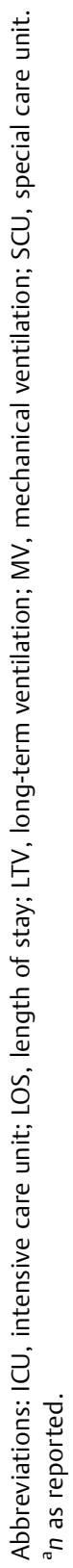


suggesting that a week or more may be needed to assess a chronic patients' response to ventilator changes.

The article by Ceriana et al was the sole international study. ${ }^{12}$ Conducted in Italy, this prospective study used a decisional flowchart to standardize decisions about timing of tracheostomy decannulation for chronic adult patients in an ICU. The flowchart included waiting periods and dictated that patients had to successfully tolerate a smaller cannula and capping for 3 to 7 days depending on the type of tracheostomy prior to decannulation. For an 18-month period, providers followed the flowchart when considering removing a tracheostomy for 145 consecutive patients. Using the flowchart, $78 \%$ of patients were able to be decannulated and only $3 \%$ required emergent reintubation in the subsequent 3 months. The decisional flowchart allowed many patients to be decannulated who had previously been determined to be "difficult to wean." Pacing was inherent to the flowchart, which consistently slowed the decannulation process by requiring days of monitoring between management changes. ${ }^{12}$

Burns et al evaluated the use of an "outcomes management" approach to weaning adult ICU patients receiving prolonged ventilation (defined as $>72$ hours). ${ }^{13}$ Outcomes management involved dedicated medical team member (advanced practice nurses) that utilized a clinical pathway that paced care and systematically targeted and tracked patient outcomes, such as length of stay (LOS), days of mechanical ventilation and cost of care. The outcomes managers and clinical pathways provided consistency in care and the pathway provided a longitudinal plan. The 124 historical controls and 181 study patients were categorized as acutely ill (Phase 1); or chronically ill if ready to wean (Phase 2); weaning (Phase 3); or in rehab (Phase 4). Patients in Phase 1 received routine ICU care while the patients in Phase 2 through 4 had a slower pace of management including less frequent laboratory evaluation which resulted in less frequent treatment manipulations. Study patients, receiving intentionally paced care, did have a nonsignificant trend to lower days of mechanical ventilation and shorter LOS with decreased costs. ${ }^{13}$

Daly et al describes the conception and initial use of a special care unit (SCU) for patients admitted to the ICU for greater than 7 days. The SCU is an eight-bed physical unit that was designed to accommodate chronic, technology-dependent ICU patients and transition to fewer ICU interventions, and increasing family involvement and rehabilitation. ${ }^{14}$ It is staffed by nurses with ICU experience who received specialized training in care of chronic patients and uses a case management and interdisciplinary care model. Patient care protocols and critical pathways standardized treatment and paced care protecting periods of rest between management changes. Patients admitted to the ICU for $>7$ days were randomly assigned to the SCU $(n=15)$ versus the traditional ICU $(n=10)$. Patients in both groups had similar mortality rates (33 vs. 30\%), but SCU patients had decreased cost and improved family and staff satisfaction. ${ }^{14}$

\section{Discussion}

As the number of chronic neonatal and pediatric ICU patients grow across the world, approaches to their care must evolve. Their unique and complex needs are not well served by current acute care models, and there are not yet enough pediatric facilities able to manage complex care outside of ICUs. Our prior hypothesis-generating qualitative work from families of children with CCI who have experienced repeated ICU populations, and from clinicians who routinely care for this population, suggests that a "slow and steady" clinical management approach which avoids rapid management changes and expects delayed treatment responses, might reduce excess ICU days. ${ }^{8}$ In theory this approach would decrease clinical setbacks by allowing time for multiorgan equilibration, which could decrease ICU days, reduce costs, and improve overall patient and family quality of life. In preparation for study design to test these theories, we performed this systematic review to summarize existing data regarding approach and pacing of chronic care delivery within ICUs.

Our most important finding was that quality data on this topic, particularly pediatric data, are sparse and of limited quality. Of all articles, none specifically addressed the question of how pace and consistency of care affected CCI patients. Studies were predominately adult focused, and many included the care of adult long-term ventilation patients who are included in the CCI patient population. Existing studies have variable definitions of patient populations, incorporate varying methodologic rigor, and target different outcomes. Using the Grading of Recommendations Assessment, Development and Evaluation (GRADE) approach, all studies had high risk of bias as shown in - Table 4, most notably for allocation concealment and blinding. Together the studies had significant imprecision. Burns et al showed a nonsignificant reduction in the duration of mechanical ventilation with an outcomes managed approach; Ceriana et al and Daly et al did not report confidence intervals or $p$-values. ${ }^{12}$ As the studies had differing

Table 4 Risk of bias

\begin{tabular}{|l|l|l|l|l|l|l|}
\hline & $\begin{array}{l}\text { Adequate } \\
\text { sequence } \\
\text { generation }\end{array}$ & $\begin{array}{l}\text { Allocation } \\
\text { concealment }\end{array}$ & Blinding & $\begin{array}{l}\text { Intention } \\
\text { to treat } \\
\text { analysis }\end{array}$ & $\begin{array}{l}\text { Incomplete } \\
\text { outcome data } \\
\text { addressed }\end{array}$ & $\begin{array}{l}\text { Free of } \\
\text { selective } \\
\text { reporting }\end{array}$ \\
\hline Burns 1998 & - & - & - & - & + & + \\
\hline Ceriana 2003 & - & - & - & - & + & + \\
\hline Daly 1991 & + & - & - & + & + & - \\
\hline Kun 2012 & n/a & n/a & n/a & n/a & + & + \\
\hline
\end{tabular}

Note: Key -+ , low risk of bias; -, high risk of bias; ?, unclear risk of bias. 
interventions, it is difficult to fully evaluate inconsistency between article results. Additionally, the evidence is indirect for the population of pediatric CCI patients as three of the four articles populations were adults. Publication bias is unclear due to the limited number of studies available. Overall these articles have a very low GRADE certainty rating. ${ }^{15}$ Nevertheless, they do offer several insights consistent with the hypothesis that a paced ("slow") and consistent ("steady") approach to chronic ICU patients' care could reduce use of ICU days and resources.

Traditional ICU management in the United States is organized around acute stabilization and early transfer to a nonICU setting. Hospitalizations of greater than 3 days in adult ICUs, and greater than 7 days in pediatric ICU's, are considered prolonged. ${ }^{16}$ Yet several days is a very modest time in the course of chronic pathophysiology, that is not "fixed" easily, if at all. All papers in this review addressed the growing subset of patients whose clinical timeframe is not hours or days but weeks or months. They also suggest that the "usual" pace of ICU care, which includes frequent treatment titrations, does not shorten ICU days. In fact, clinical practices which might otherwise prolong hospitalizations by requiring days or even weeks of observation between treatment titrations, may paradoxically shorten overall ICU days in the CCI population. This aspect deserves greater study. Some papers describe the importance that structured clinical protocols can have on pacing, by legitimizing a standard of chronic care that falls outside the typical ICU care. ${ }^{11-14}$ Some described carving out alternative inhospital locales, e.g., "special care units," where ICU-trained personnel are able to modify care plans with a slower pace. Such locations or groupings of patients may have protocols that are inherently slower paced and more broadly used providing consistency and a staff that is trained in providing continuity of care.

In addition to a slower paced plan of care, these articles suggest that consistent clinical management, in the form of protocols, improves a variety of outcomes relevant to chronic management for patients with $\mathrm{CCI}$, including duration of mechanical ventilation, LOS, and costs as well as successful decannulation. ${ }^{11-14}$ National consensus statements recognize the importance of care consistency and highlight the use of standard protocols when caring for long-term mechanically ventilated patients. ${ }^{9,10}$ Given the inherent prolonged admissions of patients with $\mathrm{CCI}$, a set protocol/plan of care prevents providers from "re-inventing the wheel" with each new team. Various adult ICU protocols are shown to streamline acute care and improve patient safety in multiple ways such as, oral care protocols leading to decreased ventilator-associated pneumonia rates, electrolyte replacement protocols reducing time from abnormal laboratory result to electrolyte normalization, and transfusion guidelines reducing the rate of nonevidencebased transfusions. ${ }^{17-21}$ While patients with $\mathrm{CCI}$ require individualized care, they also have predictable chronic needs that can be proactively managed with protocols.

Finally, any care model or intervention must account for the inherent multidisciplinary needs of these patients. A multitude of health care professionals monitor and interact with the child throughout the rest of their hospital day: the physicians, nurses, respiratory therapists, physical and occupational therapists, social workers, child life specialists, pharmacists, chaplains, and others. In the articles by Daly et al and Burns et al, a multidisciplinary team was a central tenant of intervention. ${ }^{14}$ A defining role of the SCU case manager and the outcomes manager is to coordinate the various teams involved in the patients' care. Numerous articles suggest that multidisciplinary teams improve patient care in a variety of aspects such as symptomatology, communication, and overall mortality. ${ }^{22-26}$ The papers in this review suggest that any interventions targeting pacing or consistency of chronic ICU care must be fundamentally interdisciplinary.

Capturing robust data about chronic ICU management for children with CCI patients faces several hurdles. There are variable definitions of pediatric medical complexity and CCI. ICU management for neonates versus older children incorporates different technology, medications, and staff. Outcomes of interest, such as LOS or cost, vary based on local access to non-ICU care sites (e.g., pediatric long-term care hospitals) and reimbursement structures (e.g., Maryland's Global Budget Revenue). ${ }^{27}$ Additionally, as the studies in this review demonstrate, more refined methodologies are needed to account for the many patient, family, clinician, and hospital factors that shape chronic ICU management.

Paced, consistent management for children with prolonged ICU stays merits further studies. This systematic review suggests that protocols that slow nonurgent treatment titrations and reduce nonessential interventions deserve exploration. Because most patients with $\mathrm{CCI}$ have multiorgan diseases, no single organ-specific protocol will adequately serve their needs. Yet because prolonged ICU hospitalizations are commonly due to dependence on mechanical ventilation, respiratory management strategies are an obvious initial focus for interventions. The papers in this review suggest that, even though a slow and steady approach to ventilator weaning might take multiple days/ weeks in the short term, consistency of management could ultimately shorten ICU hospitalizations. If extrapolated to pediatric $\mathrm{CCI}$ patients, they too may benefit from slower paced protocolized care with an ultimate reduction in ICU LOS stay. This mirrors the suggestions of families and clinicians in our qualitative work. ${ }^{8}$ A logical next step could be a retrospective investigation of the potential correlation between pace of care, patient setbacks, and overall LOS in pediatric CCI patients. Additional future areas of study include the location of pediatric CCI care, such as designated ICU beds or specialized units, longitudinal teams with interest and commitment to patients with $\mathrm{CCI}$, and clinician handoffs both within the ICU, between hospital units, and between facilities to promote consistent and longitudinal care.

Funding

This study was funded by the Johns Hopkins Children's Center Pediatric Innovation Award.

Conflict of Interest

None declared. 


\section{Acknowledgment}

The authors would like to thank Katie Lobner MSL for her assistance with crafting database search terms and conducting the systematic review.

\section{References}

1 Shapiro MC, Henderson CM, Hutton N, Boss RD. Defining pediatric chronic critical illness for clinical care, research, and policy. Hosp Pediatr 2017;7(04):236-244

2 Simon TD, Berry J, Feudtner C, et al. Children with complex chronic conditions in inpatient hospital settings in the United States. Pediatrics 2010;126(04):647-655

3 Edwards JD, Lucas AR, Boscardin WJ, Dudley RA. Repeated critical illness and unplanned readmissions within 1 year to PICUs. Crit Care Med 2017;45(08):1276-1284

4 Namachivayam P, Taylor A, Montague T, et al. Long-stay children in intensive care: long-term functional outcome and quality of life from a 20-yr institutional study. Pediatr Crit Care Med 2012;13 (05):520-528

5 Naghib S, van der Starre C, Gischler SJ, Joosten KFM, Tibboel D. Mortality in very long-stay pediatric intensive care unit patients and incidence of withdrawal of treatment. Intensive Care Med 2010;36(01):131-136

6 Namachivayam SP, Alexander J, Slater A, et al; Paediatric Study Group and Australian and New Zealand Intensive Care Society. Fiveyear survival of children with chronic critical illness in Australia and New Zealand. Crit Care Med 2015;43(09):1978-1985

7 van der Heide P, Hassing MBF, Gemke RJBJ. Characteristics and outcome of long-stay patients in a paediatric intensive care unit: a case-control study. Acta Paediatr 1992 2004;93(08):1070-1074

8 Henderson CM, Williams EP, Shapiro MC, et al. "Stuck in the ICU": caring for children with chronic critical illness. Pediatr Crit Care Med 2017;18(11):e561-e568

9 MacIntyre NR, Cook DJ, Ely EW Jr, et al; American College of Chest Physicians; American Association for Respiratory Care; American College of Critical Care Medicine. Evidence-based guidelines for weaning and discontinuing ventilatory support: a collective task force facilitated by the American College of Chest Physicians; the American Association for Respiratory Care; and the American College of Critical Care Medicine. Chest 2001;120(Suppl 6): 375S-395S

10 MacIntyre NR, Epstein SK, Carson S, Scheinhorn D, Christopher K, Muldoon S; National Association for Medical Direction of Respiratory Care. Management of patients requiring prolonged mechanical ventilation: report of a NAMDRC consensus conference. Chest 2005;128(06):3937-3954

11 Kun SS, Edwards JD, Ward SLD, Keens TG. Hospital readmissions for newly discharged pediatric home mechanical ventilation patients. Pediatr Pulmonol 2012;47(04):409-414

12 Ceriana P, Carlucci A, Navalesi P, et al. Weaning from tracheotomy in long-term mechanically ventilated patients: feasibility of a decisional flowchart and clinical outcome. Intensive Care Med 2003;29(05):845-848
13 Burns SM, Marshall M, Burns JE, et al. Design, testing, and results of an outcomes-managed approach to patients requiring prolonged mechanical ventilation. Am J Crit Care 1998;7(01):45-57, quiz 58-59

14 Daly BJ, Rudy EB, Thompson KS, Happ MB. Development of a special care unit for chronically critically ill patients. Heart Lung 1991;20(01):45-51

15 Schünemann H, Brożek J, Guyatt J, Oxman A. GRADE Handbook for Grading Quality of Evidence and Strength of Recommendations. Updated October 2013. The GRADE Working Group. Available at: https://gdt.gradepro.org/app/handbook/handbook.html

16 Marcin JP, Slonim AD, Pollack MM, Ruttimann UE. Long-stay patients in the pediatric intensive care unit. Crit Care Med 2001;29(03):652-657

17 Borges LGA, Savi A, Teixeira C, et al. Mechanical ventilation weaning protocol improves medical adherence and results. J Crit Care 2017;41:296-302

18 Lee Y-LL, Sims KD, Butts CC, et al. The combination of SAT and SBT protocols may help reduce the incidence of ventilator-associated pneumonia in the burn intensive care unit. J Burn Care Res 2017; 38(02):e574-e579

19 Ory J, Raybaud E, Chabanne R, et al. Comparative study of 2 oral care protocols in intensive care units. Am J Infect Control 2017;45 (03):245-250

20 Seitz KP, Sevransky JE, Martin GS, Roback JD, Murphy DJ. Evaluation of RBC transfusion practice in adult ICUs and the effect of restrictive transfusion protocols on routine care. Crit Care Med 2017;45(02):271-281

21 Pearson DJ, Sharma A, Lospinoso JA, Morris MJ, McCann ET. Effects of electrolyte replacement protocol implementation in a medical intensive care unit. J Intensive Care Med 2018;33(10):574-581

22 Brilli RJ, McClead RE Jr, Crandall WV, et al. A comprehensive patient safety program can significantly reduce preventable harm, associated costs, and hospital mortality. J Pediatr 2013; 163(06):1638-1645

23 Shaw DJ, Davidson JE, Smilde RI, Sondoozi T, Agan D. Multidisciplinary team training to enhance family communication in the ICU. Crit Care Med 2014;42(02):265-271

24 Collinsworth AW, Priest EL, Campbell CR, Vasilevskis EE, Masica AL. A review of multifaceted care approaches for the prevention and mitigation of delirium in intensive care units. J Intensive Care Med 2016;31(02):127-141

25 Fanari Z, Barekatain A, Kerzner R, Hammami S, Weintraub WS, Maheshwari $\mathrm{V}$. Impact of a multidisciplinary team approach including an intensivist on the outcomes of critically ill patients in the cardiac care unit. Mayo Clin Proc 2016;91(12):1727-1734

26 Marra A, Ely EW, Pandharipande PP, Patel MB. The ABCDEF bundle in critical care. Crit Care Clin 2017;33(02):225-243

27 Boss RD, Williams EP, Henderson CM, et al. Pediatric chronic critical illness: reducing excess hospitalizations. Hosp Pediatr 2017;7(08):460-470

28 Moher D, Liberati A, Tetzlaff J, Altman DG; PRISMA Group. Preferred reporting items for systematic reviews and meta-analyses: the PRISMA statement. PLoS Med 2009;6(07):e1000097 